\title{
Estrategias Pedagógicas en Procesos de Enseñanza y Aprendizaje en la Educación Superior incluyendo Tecnologías de la Información y las Comunicaciones
}

\author{
Madelin Sánchez-Otero ${ }^{1}$, Jesús García-Guiliany ${ }^{2}$, Ernesto Steffens-Sanabria ${ }^{3}$ y Hugo Hernández- Palma ${ }^{4}$ \\ (1) Universidad de la Costa, Dpto. de Gestión Organizacional, Calle 58 \# 55-24. Barranquilla-Colombia \\ (2) Universidad Simón Bolívar, Fac. Administración y Negocios, Calle 59 \#59-81. Barranquilla-Colombia \\ (3) Corp. Universitaria Latinoamericana, Fac. Ciencias Económicas, Calle 58 \# 55-24A Barranquilla-Colombia \\ (4) Universidad del Atlántico, Fac. Ciencias Económicas, Cra 30 \# 8- 49. Puerto Colombia -Colombia \\ (e-mail: msanchez6@cuc.edu.co; jesus.garcia@unisimonbolivar.edu.co; steffensse@ul.edu.co; \\ hugohernandezp@mail.uniatlantico.edu.co)
}

Recibido Jun. 19, 2018; Aceptado Ago. 17, 2018; Versión final Oct. 3, 2018, Publicado Jun. 2019

\begin{abstract}
Resumen
El objetivo del presente artículo es analizar cómo se han transformado las estrategias pedagógicas en la educación superior con la inserción de las Tecnologías de la Información y las Comunicaciones (TICs) en los últimos años. Mediante un enfoque cualitativo y una revisión documental exhaustiva se realizó un detallado análisis de publicaciones desde el año 2000 en adelante. Los hallazgos sugieren que en los últimos años se ha visto un importante avance de la tecnología en todos los aspectos cotidianos. Adicionalmente, se evidenciaron aportes significativos que muestran como en Colombia y otros países, los escenarios educativos han cambiado sustancialmente gracias a las tecnologías que hoy día se aplican para apoyar el proceso de enseñanza y aprendizaje. Las conclusiones se orientan a que la difusión de estrategias pedagógicas en procesos de enseñanza-aprendizaje mediados por las TIC, fortalece significativamente la labor docente.
\end{abstract}

\section{Pedagogical Strategies in Teaching and Learning Processes in Higher Education including Information and Communication Technologies}

\begin{abstract}
The objective of this article is to analyze how pedagogical strategies in higher education have been transformed by the inclusion of Information and Communication Technologies (ICTs) in recent years. By means of a qualitative approach and an exhaustive documentary review, a detailed analysis of publications since the year 2000 was carried out. The findings and analyses suggest that in recent years there has been an important advance of technology in all aspects of daily life. Additionally, significant contributions were found that show how in Colombia and other countries, the educational scenarios have changed substantially thanks to the technologies that are nowadays applied to support teaching and learning processes. The main conclusion is that the dissemination of pedagogical strategies in teaching-learning processes using ICTs significantly strengthens the teaching work.
\end{abstract}




\section{INTRODUCCIÓN}

Desde mediados de los años 80 se empezó a hablar de tecnologías aplicadas a la información, adoptando diferentes definiciones durante décadas, siendo en el siglo XXI cuando se empieza a reconocer por algunos autores e instituciones el papel fundamental de las TIC en la sociedad (Vila et al., 2016). La mayoría de las definiciones usadas asocian un componente tecnológico y su impacto en la gestión de la información (Cammaerts, 2017). Se entienden entonces las TIC como las herramientas tecnológicas digitales que facilitan la comunicación y la información, con potencial para mejorar la sociedad y sus desarrollos pueden tener fines económicos (Causado et al., 2015). Las TIC poseen como características la inmaterialidad, interactividad, instantaneidad, innovación, elevada calidad en imagen y sonido, digitalización e interconexión (Jin y Cho, 2015). La presencia de las TIC en las actividades sociales y productivas del individuo actual, han llevado a desarrollar todo un mundo alrededor de la generación de información masiva, interacción de comunidades, integración comercial, comunicaciones electrónicas, noticias, formación, investigación, desarrollo científico e innovación entre otros aspectos (Arkorful y Abaidoo, 2015). En Colombia existe un gran apoyo del gobierno hacia las TIC, impulsado por la creación en 2009 del de Ministerio de Tecnologías de la Información y las Comunicaciones (MINTIC), antiguo Ministerio de Comunicaciones, para la promoción del acceso y uso de las TIC, así como para el desarrollo de las iniciativas necesarias para dar un salto en cobertura, expansión y superación de las barreras en pos de las proyecciones trazadas desde la directriz estatal (Duarte et al., 2016).

En su labor, el MINTIC ha reconocido la importancia de llevar las TIC a las aulas, impulsando iniciativas como la dotación de las aulas de clase de colegios oficiales con tabletas digitales y computadores, como estrategia de integración de toda la población. Esto ha logrado explorar nuevos métodos de enseñanza y alternativas de capacitación que se extienden también al cuerpo docente (Cifuentes, 2016). Ante este impulso de iniciativas de llevar las TIC a los ámbitos escolares, diversos autores señalan que la necesidad de articular la sociedad y la tecnología preparando a las instituciones de educación superior para el desarrollo, capacitación e inserción de nuevas tecnologías como el internet de las cosas, como un factor diferenciador que puede impulsar notoriamente los resultados a futuro (Cifuentes, 2015). Según la Unión Internacional de Telecomunicaciones (UIT), agencia especializada de la Organización de las Naciones Unidas (ONU) para las TIC (2018), es de amplio reconocimiento la importancia de estas. Para el año 2030 se ha trazado una agenda de desarrollo sostenible que reconoce que la difusión de la información y tecnologías de comunicación y la interconexión global tiene un gran potencial de acelerar el progreso, cerrando la brecha de lo digital y desenvolviendo sociedades de conocimiento para los países menos desarrollados (Rajabi et al., 2014).

En el informe sobre la medición de la sociedad de la información de la UIT (2015), ofrece una visión global de los últimos avances de las TIC y se presenta el comportamiento del Índice de Desarrollo de las TIC (IDT). En dicho informe se puede ver el aumento considerable que ha tenido en los últimos años el acceso a la utilización de las TIC. EI IDT es un índice que contempla 11 indicadores que arrojan una medida de referencia que permite analizar la evolución de las tecnologías bajo los parámetros de nivel de de sarrollo y evolución en el tiempo de desglose de las TIC, progresos alcanzados, brecha entre países según su nivel y potencial de desarrollo (Rajabi et al., 2014). Los resultados arrojados por el informe muestran que entre 2010 y 2015 todos los países aumentaron su IDT, siendo la república de Corea quien obtiene el mejor índice. Las 10 principales economías con el mejor índice corresponden en su mayoría a países europeos de altos ingresos, reflejando una alta correlación entre el IDT y el ingreso nacional per cápita. Colombia se ubicó en el 2010 en el puesto 83 y en el 2015 en el puesto 75 dentro del ranking mundial (Iglesias et al., 2015).

Según cifras del MINTIC, la evolución del uso de TIC ha ido en aumento en los últimos años, mostrando una tendencia en Colombia acorde al crecimiento de uso de internet, plataforma primordial para uso de tecnologías TIC y representando el principal medio de interacción con base tecnológica. Esto ha impactado diversos sectores de la sociedad y la forma en las que tradicionalmente interactúan las personas, en particular a la educación, donde los procesos de enseñanza y aprendizaje tienen la oportunidad para mejorarse y transformarse conforme a estos avances; las figuras 1 y 2 muestran indicadores relacionados con la realidad citada. En función de todo lo expuesto, el objetivo del presente artículo se orienta a analizar el impacto que ha tenido en la educación superior la inserción de las Tecnologías de la Información y las Comunicaciones (TICs) en los últimos años, fundamentado en la reflexión sobre cómo se han transformado las estrategias pedagógicas en el entorno del aula de clase, la interacción entre alumno docente y las competencias necesarias en los profesionales de la educación para el manejo de estas nuevas tecnologías en los procesos de enseñanza y aprendizaje para su máximo aprovechamiento. 


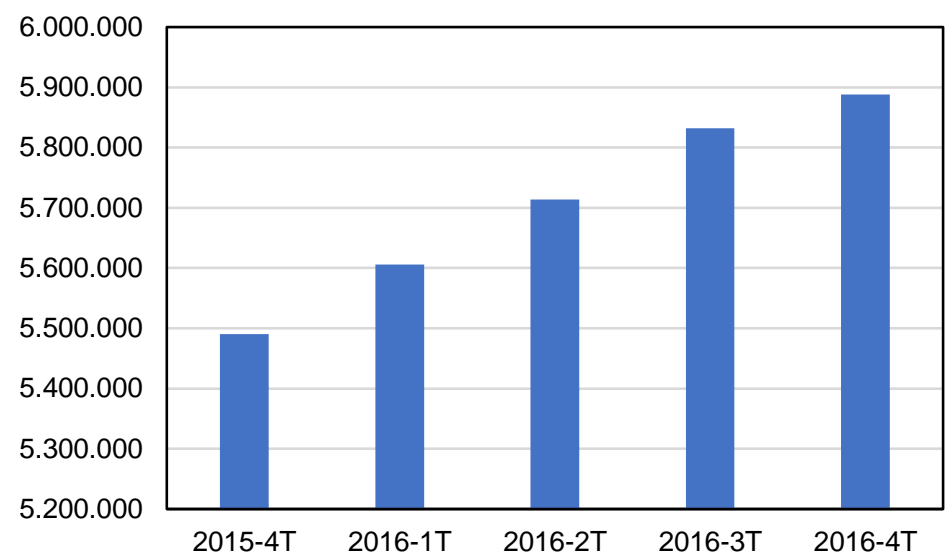

Fig. 1: Crecimiento de usuarios en banda ancha en Colombia (Datos tomados de MINTIC, 2017)

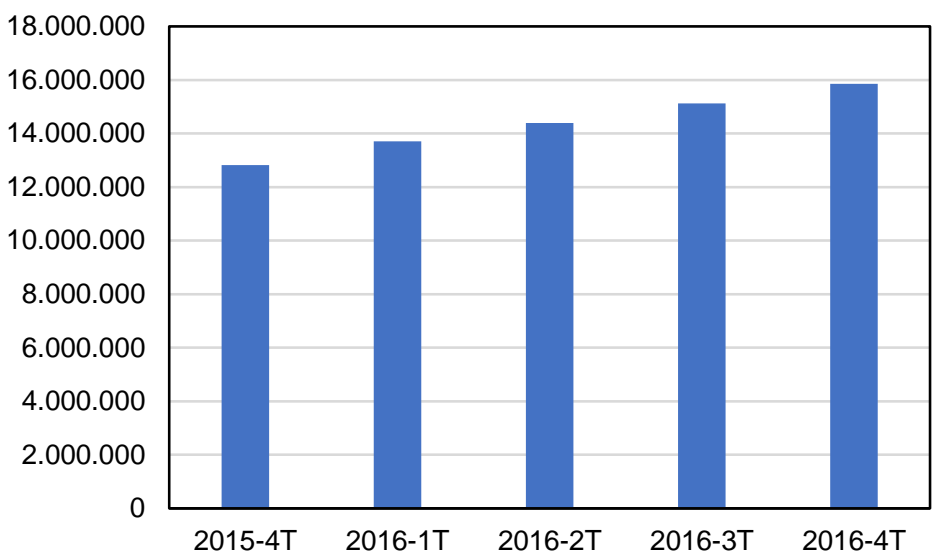

Fig. 2: Evolución de suscripción al servicio de internet (Datos tomados de MINCIT, 2017)

\section{BASES TEÓRICAS}

Uno de los principales fundamentos teóricos necesarios para comprender los planteamientos aquí tratados, gira entorno a las estrategias pedagógicas en el proceso de enseñanza y aprendizaje. En virtud de ello se exponen algunos referentes sobre el tema con la finalidad de dar un marco de interpretación a los resultados y hallazgos del estudio. Para ello, se realiza una revisión de los postulados y planteamientos de los autores en la literatura científica, sobre todo aquella relacionada con los ambientes educativos de educación superior, dando una perspectiva desde las TIC.

\section{Estrategias pedagógicas}

Para llevar a cabo el proceso de enseñanza y aprendizaje, el docente cuenta con diversidad de herramientas que le permite interactuar con los alumnos para fomentar su participación, motivación e interés por el tema tratado, con la finalidad de transmitir el conocimiento que posee de una manera significativa (Torres y Velandia, 2017). Este planteamiento, necesariamente implica una formación docente robusta capaz de traducir los conocimientos en aprendizaje significativo en sus estudiantes (DeMonte, 2013), en este sentido, el diseño de estrategias para la enseñanza debe ir orientadas a un propósito específico tomando en cuenta las necesidades y características del grupo. En el ámbito de las teorías pedagógicas, diversidad de autores han desarrollados sus visiones sobre las estrategias que debe desarrollar el docente en su práctica educativa, concretamente, orientadas hacia el proceso de enseñanza y aprendizaje. El enfoque tradicional, se enfoca particularmente en el docente, pues es quien realiza la exposición de los contenidos y diseña e implementa las actividades en el aula de clase; en este escenario, la participación del estudiante queda relegada a una posición pasiva de recepción de instrucciones y conocimiento (Sikoyo, 2010). Esta concepción cambió con la introducción de las ideas de la escuela nueva (Ferreire, 1953) la cual postula un mayor interés y respecto por las demandas educativas del estudiante, basado fundamentalmente en la educación para la libertad.

Otras visiones sobre la pedagogía, fueron expuestas por Coll et al. (2001) respecto a las teorías constructivistas y conductivistas, quien integró la psicología para dar respuesta desde este ámbito a las 
preguntas que se planteaban en ese entonces desde la pedagogía. En este mismo orden de ideas, surgió la llamada pedagogía conceptual, la cual se enfoca en la enseñanza para la adquisición de competencias para que los estudiantes logren empoderarse de forma positiva de sus procesos de aprendizaje (Igwebuike y Oriaifo, 2012). Con base en lo expuesto, la evolución de perspectivas teóricas sobre la pedagogía y las estrategias que se utilizan para su desarrollo, están siendo transformadas por la incorporación de la tecnología en los procesos de enseñanza (Ali et al., 2013).

\section{Tecnologías de la Información y la Comunicación}

Los avances tecnológicos impulsados por los desarrollos en el área de la computación, están cambiando de forma vertiginosa el estilo de vida en la sociedad actual; por ende, la educación superior, es sin duda uno de los sectores que está realizando esfuerzos por innovar en sus procesos educativos para dar respuesta a estas demandas con la incorporación de las TIC en sus procesos de enseñanza, sin embargo, la amplitud de herramientas que se consideran como parte de esta categoría, requiere de algunas precisiones teóricas para establecer de forma concreta qué son y cómo pueden aplicarse. En términos generales, resulta complejo establecer una definición única sobre las TIC, entendiendo que poseen características y propósitos diversos; sin embargo, es posible hacer una aproximación conceptual desde un punto de vista educativo, afirmando que son un conjunto de herramientas y recursos que estimulan y contribuyen a la interacción dinámica entre individuos facilitando así el aprendizaje y la divulgación de los saberes (Vasović y Milašinović, 2014). Desde esta perspectiva, algunos autores han planteado una profundización sobre la concepción que se tiene de las TIC respecto a la incorporación en los procesos educativos, las cuales pueden darse desde tres ópticas, las TIC propiamente, las tecnologías para el aprendizaje y conocimiento (TAP) y las tecnologías para el empoderamiento y la participación (TEP) (Cabero, 2015).

Cada uno de estos enfoques sobre la tecnología aplicada en la enseñanza y aprendizaje, cumple un rol distintivo, lo cual permite comprender que el alcance y propósito de estas herramientas no es necesariamente el mismo. En cuanto a las TAP, se puede decir que facilitan de forma importante el aprendizaje por medio del análisis del contexto en el que se desenvuelve el estudiante (Rodríguez, 2016). Por su parte, las TEP se enfocan hacia la participación y colaboración entre los actores del proceso educativo, comprendiendo que el aprendizaje no es individual, sino que cuenta con una dimensión social para construir conocimiento, tal como lo sostiene la corriente Vygotskiana (Churcher, 2014).

\section{METODOLOGÍA}

El desarrollo del presente artículo se orientó metodológicamente en el enfoque cualitativo con base en una revisión documental, el cual ha sido utilizado en investigaciones similares en el campo educativo (Owen, 2014). La aproximación al objeto de estudio por medio este método permite dar flexibilidad en cuanto a la comprensión de la realidad analizada desde la perspectiva de las ciencias sociales, dado que no se realizan con base en interpretaciones numéricas o datos estadísticos propios del paradigma positivista (Ritchie, et al., 2013). Por otra parte, se utilizó la revisión documental para aproximarse al tema de estudio; al respecto, Hartas (2015) señala que este tipo de técnica permite obtener múltiples perspectivas del tópico estudiado, así como también ofrece un punto de referencia teórico, funcionando como un recurso significativo en los enfoques multi método que requieren triangulación de información.

Por lo citado, la investigación se apoya en la consulta de estudios previos que hayan tenido por objeto central, analizar las TIC y su impacto en la educación superior, tomando como patrón de selección de la información que dichos documentos se hayan publicado en bases de datos de reconocimiento nacional e internacional como: Scielo, Elsevier, Emerald, Springer y similares; aspectos como propósito del estudio y aportes al tema actual fueron contrastados de manera organizada. En este sentido, se utilizaron palabras clave para realizar la revisión documental tales como: tecnología de la información y comunicación, educación superior, proceso educativo, proceso de enseñanza y aprendizaje, procesos pedagógicos, pedagogía, estrategias pedagógicas, competencias docentes en TIC y tecnologías emergentes en el aula de clases, que dieran cuenta de los artículos científicos relacionados con el tema.

A partir de la búsqueda de documentos, se organizaron sistemáticamente en una matriz de elaboración propia, en la cual se colocaron datos relacionados con autores, título, año, objetivos, resultados y principales aportes, con la finalidad de poder determinar si los hallazgos de las investigaciones contribuían de forma importante con el objetivo propuesto. En total se revisaron cerca de 50 documentos basados en los siguientes criterios de búsqueda: a) publicaciones a partir del año 2000, exceptuando autores y teorías clásicas; b) mayor número de artículos en idioma inglés de preferencia, sin descartar aquellos relevantes en idioma español; c) mayormente publicados en revistas de corriente principal. 


\section{RESULTADOS Y DISCUSIÓN}

La información encontrada ha sido agrupada en dos grandes áreas para una mejor discusión: i) el uso de la TICs en el proceso educativo; y ii) las TICs en el proceso pedagógico universitario.

\section{Las TIC en el proceso educativo}

Los sistemas educativos no han sido ajenos a la evolución de las TIC, que requieren de ellas constantes renovaciones a partir de la relación tecnología-sociedad, que a su vez determina la relación tecnologíaeducación (Hunsu et al., 2016). La sociedad actual requiere del sistema educativo la implementación de procesos y estrategias pedagógicas mediadas por herramientas tecnológicas, que brinden al docente la posibilidad de aplicar procedimientos organizados para llevar al estudiante a concentrar su atención en el aprendizaje, resaltando la importancia en la incorporación de estrategias mediadas por TIC de manera efectiva (Marín et al., 2017).

Esta implementación conlleva numerosos cambios a nivel de infraestructura tecnológica; a nivel de profesorado, cuyo rol cambia de transmitir contenidos al de estimular la búsqueda de conocimiento por parte del alumno; y cambios por parte del alumno al pasar a involucrarse en procesos de discusión, negociación e interacción con su grupo en actividades de colaboración e intercambio de opiniones (Morales et al., 2015). Autores diversos coinciden en plantear que la naturaleza de las TIC, al buscar mejoras en los procesos de procesamiento de datos y distribución de la información, resulta de gran provecho para su utilización en el contexto educativo, ofreciendo grandes ventajas en el proceso de enseñanza-aprendizaje al favorecer la reducción de tiempos y distancias en el intercambio de información. Sin embargo, para su éxito necesita interactuar con todos los elementos involucrados en el proceso de educativo como son: objetivos, contenidos, profesores, alumnos y directivos como se muestra en la figura 3 (Drachsler et al., 2015).

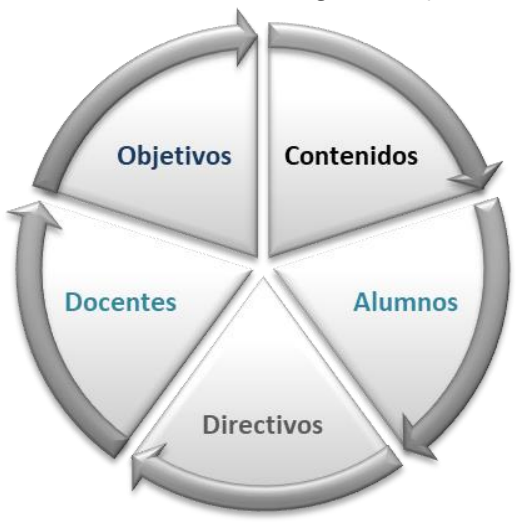

Fig. 3: Elementos relevantes en el proceso educativo (Adaptada de Drachsler et al., 2015)

De igual forma, otros investigadores han enunciado, que las políticas gubernamentales en Latinoamérica hasta hace poco tiempo se centraban en la dotación de hardware en las escuelas, y la clave radica en los contenidos y servicios a los que se puede tener acceso como apoyo a la educación, siendo las TIC una herramienta para ello, destacando como un acierto la apuesta a la capacitación de la comunidad educativa en temas de tecnología. En esto radica la importancia de las TIC y el motivo de su inclusión en la agenda educativa de los gobiernos (Vargas, 2016). Las TIC aportan a la ampliación de la oferta informativa, creando entornos flexibles para el aprendizaje, eliminando barreras espacio-temporales, incrementando las modalidades comunicativas, potenciando escenarios y entornos interactivos, favoreciendo el aprendizaje y autoaprendizaje, creando opciones de tutoría y facilitando la formación permanente. Su aplicación motiva al estudiante al captar su atención y lo lleva a ser uno de los motores de su propio aprendizaje. Por lo anterior, es imprescindible tener un nivel de alfabetización digital tanto en docentes como en alumnos, para asegurar el éxito del uso de las diferentes herramientas las que se tiene disponibilidad en la era actual (Martelo et al., 2016).

Las primeras herramientas electrónicas que llegaron a las aulas fueron los medios audiovisuales. El uso de los proyectores y diapositivas abrieron el campo para el uso de tecnología en los procesos de transmisión de conocimiento en el aula de clases, y propiciaron los primeros cambios o refrescamientos en la manera como se abordaban las aulas para ser estimuladas de manera positiva. Al integrase el uso de las TIC, se permite hablar en términos de inclusión, consintiendo acoger a alumnos con necesidades educativas diversas que antes habían sido excluidos, logrando adaptar la enseñanza y la escuela a las necesidades del alumno. Esto logra que la inserción y expansión de las TIC en la educación responda a las necesidades de toda la población educativa, potencializando su participación en el proceso de enseñanza-aprendizaje, reduciendo barreras de exclusión al eliminar las limitaciones de tiempo y espacio al estar dispersa la información (Said-Hung et al., 2015). 
Sumado a todo lo tratado, es preciso afirmar que la evolución de las tecnologías permite a hoy identificar ocho dimensiones en las cuales se debe tener algún grado de competencia para poder aprovechar los beneficios de estas herramientas en los procesos educativos dentro del aula por parte del docente. En este sentido Prendes (2010) compiló los indicadores más utilizados para medir estas competencias docentes en TIC a nivel global. La figura 4 destaca cada uno de los ocho bloques identificados por el autor para la incorporación de las herramientas tecnológicas en el proceso de enseñanza y aprendizaje.

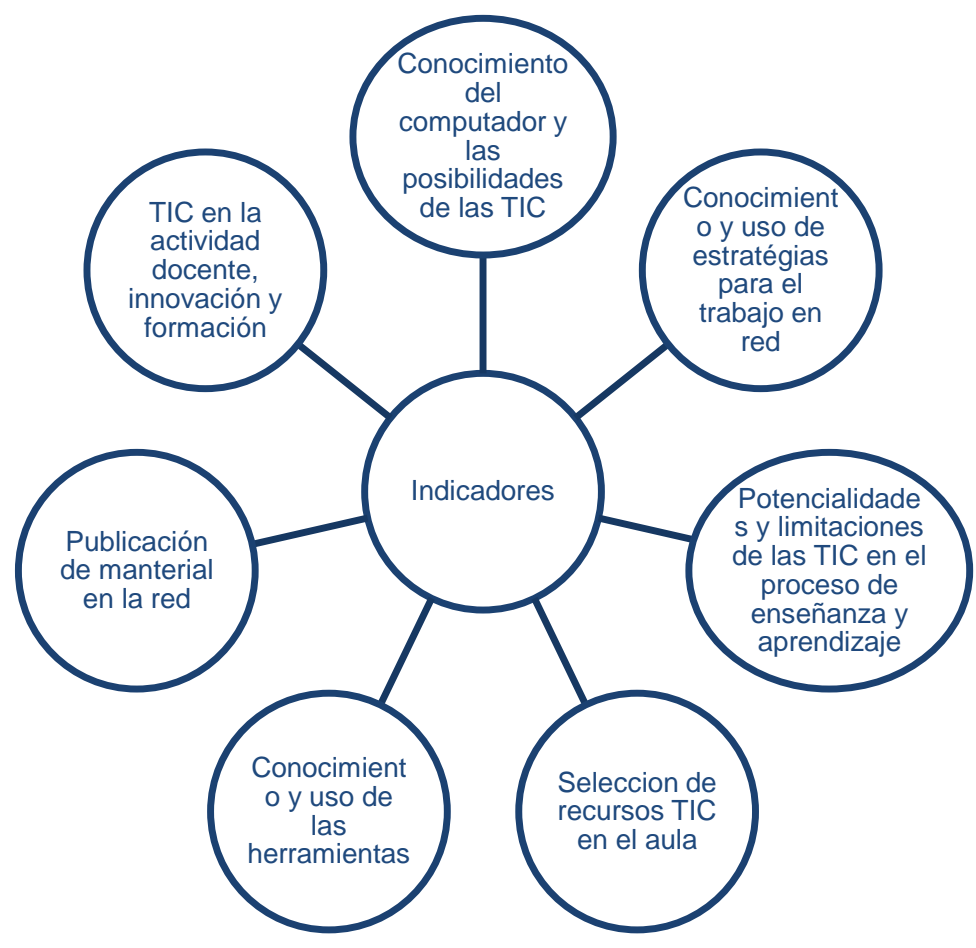

Fig. 4: Indicadores para la medición de competencias TIC (Adaptada de Prendes, 2010)

Por otra parte, García, et al. (2016) plantea que para articular las TIC en el aula se requiere analizar los principios de la integración curricular de los medios tecnológicos para como herramientas didácticas. En esto se debe tener en cuenta el entorno cultural de los estudiantes, de manera que facilite el aprendizaje, desarrollo de habilidades y competencias, dentro de un contexto digital. Es importante también la formación de los catedráticos de manera constante para promover al docente en el uso de las TIC. Para lo enunciado, se pueden tener en cuenta 3 dimensiones de formación docente: conocimientos y competencias sobre TIC como recursos didácticos en aulas presenciales y virtuales; conocimientos del currículo oculto; y conocimientos de los contextos de la realidad escolar y social. Estos puntos presentan un proceso integral de concientización docente de la importancia de las TIC (Niebles-Núñez, et al., 2016), ver figura5 con la relación detallada.

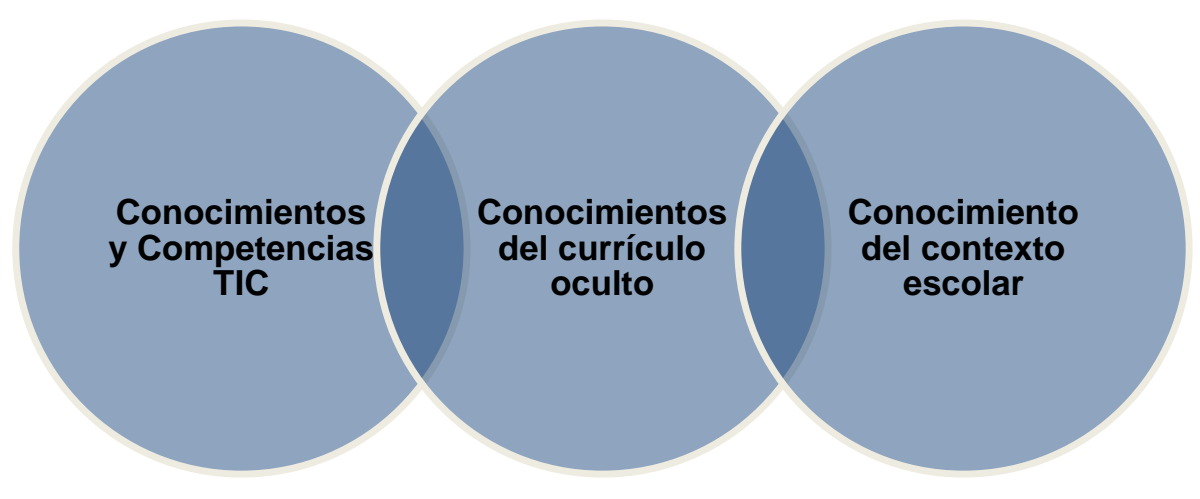

Fig. 5: Dimensiones de Formación del docente en TIC (Adaptada de Niebles-Núñez, et al., 2016)

Al ser las tecnologías una herramienta que cambia continuamente, el proceso de capacitación docente debe ser también continuo y de renovación periódica, integrando la tecnología en su proceso de formación, de manera que se asegure su competencia en TIC y así tener la capacidad de orientar a sus educandos, al 
mismo tiempo que se da una construcción y comprensión del papel que juegan éstas en la sociedad actual, facilitando el proceso de enseñanza y beneficiando a los alumnos en la ubicación de información y dedicación de tiempo a sus actividades (Ersoy y Bozkurt, 2015).

\section{TIC en el proceso pedagógico universitario}

Las entidades de educación superior juegan un papel relevante en la sociedad, al requerir de ellas e ir más allá de ser un lugar de enseñanza y en cambio ser un lugar de actualización, educación continuada, aplicando estrategias de educación funcionales, comprometidas con la investigación y su aporte a la solución de los problemas actuales. Hoy las demandas de las comunidades, exigen que las instituciones de educación superior sean incluyentes y faciliten participar en los ambientes políticos, económicos, culturales, ambientales y sociales, formando profesionales con hábitos de innovación, producción y transformación apoyados en la ciencia y la tecnología (Valencia, 2015). El proceso de enseñanza-aprendizaje es la interacción entre los elementos docente, estudiante, contenido, estrategias, medio, contexto y ambiente. Las estrategias didácticas son las acciones, estructuras o pasos planeados por el maestro para desarrollar acciones buscando propiciar la formación y lograr la meta de aprendizaje. Actualmente se promueve un modelo que se centra en el alumno, por lo que el docente diseña las actividades, enseña a aprender y evalúa, mientras que el alumno realiza las actividades, construye su propio aprendizaje y se autoevalúa (Herrera, 2015). El objetivo actual de las TIC es el logro de mejores aprendizajes que despierten el interés de los estudiantes a través de diferentes estrategias, que utilicen estas herramientas y el papel del docente sea el de orientador y liderar, más el papel protagónico recae en el estudiante como tal.

Las estrategias sirven para el mejoramiento de la calidad y el rendimiento de los alumnos, apoyadas en concepciones de aprendizaje. Para su construcción se propone tener en cuenta elementos de independencia, supervisión, autodirección y evaluación, de manera que permitan ser modificables de manera flexible y acorde a los medios y contextos donde serán aplicadas y se sugieren sean organizadas de acuerdo con su finalidad. Deben ser seleccionadas bajo criterios de validez, variedad, relevancia, claridad, adecuación, conocimiento e inserción (Ausín et al., 2016). Existen numerosas herramientas virtuales de las cuales hacer uso en el proceso educativo, con nutridas ventajas y cualidades para llevar el proceso de enseñanza centrado en el alumno. Las nuevas formas de educación que surgen a partir del uso de las TIC han dado origen al electronic learning (e-learning), como una plataforma de educación a distancia, permitiendo llegar el proceso educativo a cualquier parte del mundo sin importar las fronteras. Este tipo de herramienta puede apoyarse en otros dispositivos electrónicos que puedan proporcionar información multimedia. Del e-learning se ha dado origen al móvil learning (m-learning), que facilita el envío y recepción de información mediante cualquier dispositivo móvil; el video learning (v-learning), que se apoya en el uso de gráficos, animaciones y personajes no reales; $y$ el blended learning (b-learning), que es un aprendizaje combinado para ser bimodal, semipresencial $o$ semivirtual, en una plataforma de internet con varias herramientas como chat, foros, wikis, blogs, teleconferencias, entre otros (Valencia, 2015).

\section{CONCLUSIONES}

Las reflexiones finales dejan entrever, en concordancia con lo expuesto por Valencia (2015) que la difusión de estrategias pedagógicas en procesos de enseñanza-aprendizaje mediados por las TIC, se pueden enfocar en todos los centros de formación, fortaleciendo la educación del docente para tal fin, pues es este la persona que puede dinamizar las estrategias de aprendizaje que posibilitarán avances diferenciales en los diferentes niveles de educación. Sin embargo, dichas estrategias deben enfocarse de acuerdo a las teorías y tendencias actuales sobre pedagogía, dado los hallazgos en áreas como la neurociencia cognitiva está mostrando un nuevo camino para la transformación de los modelos educativos tradicionales (Boone y Piccinini, 2016). Como lo han propuesto Morales et al. (2015) y Steffens et al. (2017) se encuentra que la actitud e integración de los alumnos y profesores que hagan parte de los procesos apoyados en TIC se tornará positiva si logran alinear sus intereses. Por esto recomiendan que el desarrollo profesional del docente sea incrementado, para optimizar el manejo de las TIC y sacar su máximo provecho. En este aspecto, la formación docente en las instituciones de educación superior, debe tomar en cuenta el desarrollo de competencias para el manejo de las TIC en el currículo de la carrera profesional, con la finalidad de garantizar que los egresados cuenten con las habilidades necesarias para implementar estas herramientas en los procesos de enseñanza.

EI uso de TIC por parte del docente debe darse de manera cotidiana, para fortalecer sus debilidades en la acogida adecuada de las mismas y promover la renovación del proceso de enseñanza. La necesidad de cambio de metodología tradicional a una más activa es innegable, en la que el alumno sea más activo en su participación y responsable de su propio aprendizaje, lo cual con la implementación de las TIC en el aula se hace más fácil de alcanzar con la dinámica que logre insertar el docente. El reto frente a este escenario, involucra el esfuerzo y compromiso de docente, institución y alumno, en donde cada uno desde su posición aporte de manera integral al proceso de enseñanza y aprendizaje. En el contexto de la educación superior, 
estos desafíos son aún mayores, comprendiendo que la diversidad de modalidades educativas que se desarrollan actualmente, aunado a las nuevas formas para impartir el conocimiento, tales como e-learning, m-learning, v-learning y b-learning, ponen de manifiesto los cambios profundos que requiere el sistema educativo para dar respuesta a las necesidades educativas del estudiante y profesional moderno.

\section{REFERENCIAS}

Ali, G., F. Haolader y K. Muhammad, The Role of ICT to Make Teaching-learning Effective in Higher Institutions of Learning in Uganda, International Journal of Innovative Research in Science, Engineering and Technology, 2(8), $4061-4073$ (2013)

Arkorful, V. y N. Abaidoo, The Role of e-learning, Advantages and Disadvantages of its Adoption in Higher Education, International Journal of Instructional Technology and Distance Learning, 12(1), 29-42 (2015)

Ausín, V., V. Abella, V. Delgado y D. Hortigüela, Aprendizaje Basado en Proyectos a través de las TIC: Una Experiencia de Innovación Docente desde las Aulas Universitarias, Formación Universitaria, 9(3), 31-38, (2016)

Boone, W. y G. Piccinini, The Cognitive Neuroscience Revolution, Synthese, 193(5), 1509-1534 (2016)

Cabero, A., Reflexiones Educativas sobre las Tecnologías de la Información y la Comunicación (TIC), Tecnología, Ciencia y Educación, 1, 19-27 (2015)

Cammaerts, B., ICT-usage Among Transnational Social Movements in the Networked Society: To Organise, to Mobilise and to Debate. In Media, Technology and Everyday Life in Europe, 71-90, Routledge (2017)

Causado, E., J. García, J. Martínez y A. Herrera, Tecnologías de Información y Comunicación en el Sector Hotelero, Colombia, Editorial Corporación Universitaria (2015)

Churcher, K., "Friending" Vygotsky: A Social Constructivist Pedagogy of Knowledge Building through Classroom Social Media Use, Journal of Effective Teaching, 14(1), 33-50 (2014)

Cifuentes, G. A., Conceptualizando Prácticas de Liderazgo de las TIC: Un Estudio en la Educación Superior Colombiana, Education Policy Analysis Archives / Archivos Analíticos de Políticas Educativas, 24 (2016)

Cifuentes, G., Liderazgo de las TIC en Educación Superior: Estudio de Caso Múltiple en Colombia, Comunicar, 23(45), 133-142 (2015)

Coll, C., J. Palacios y A. Marchesi, Desarrollo Psicológico y Educación, Psicología de la Educación Escolar, Madrid, Alianza (2001)

DeMonte, J., High-Quality Professional Development for Teachers: Supporting Teacher Training to Improve Student Learning, Center for American Progress, 28 (2013)

Drachsler, H., K. Verbert, O. C. Santos y N. Manouselis, Panorama of Recommender Systems to Support Learning, In Recommender systems handbook, 421-451, Springer, Boston, MA (2015)

Duarte, M.A., A. A. Suárez y C. A. Suárez, Políticas y Programas del Sistema Educativo Colombiano como Marco para la Articulación de las TIC, Revista ALETHEIA, 8(1), 12-31 (2016)

Ersoy, A. y M. Bozkurt, Understanding an Elementary School Teachers' journey of Using Technology in the Classroom from Sand Table to Interactive Whiteboard, International Electronic Journal of Elementary Education, 8(1), 1 (2015)

Ferreire, A., La Escuela sobre Medida, a la Medida del Maestro, Buenos Aires, Kapelusz (1953)

García, A., M. Libedinsky, P. Pérez y M. García (Coords.), Las TIC en la Escuela Secundaria. Proyectos, Consejos y Herramientas para la Inclusión Digital, Praxis Educativa, 20(2), 70-72 (2016)

Hartas, D., Educational Research and Inquiry: Qualitative and Quantitative Approaches, Bloomsbury Publishing (2015)

Herrera J., Una Mirada Reflexiva sobre las TIC en Educación Superior, Revista electrónica de investigación educativa, 17(1), 1-4 (2015)

Hunsu, N.J.,O. Adesope y D. J. Bayly, A Meta-analysis of the Effects of Audience Response Systems (clicker-based technologies) on Cognition and Affect, Computers and Education, 94, 102-119 (2016)

Iglesias, E., L. Cano y A. García, Informe anual del Índice de Desarrollo de la Banda Ancha en América Latina y el Caribe, IDBA 2014, Inter-American Development Bank (2015)

Igwebuike, T. y S. Oriaifo, Nature of Classroom Environment and Achievement in Integrated Science: A Test of Efficacy of a Constructivist Instructional Strategy, International Journal of Research Studies in Educational Technology, 1(2), 17-29 (2012)

Jin, S. y C.M. Cho, Is ICT a New Essential for National Economic Growth in an Information Society? Government Information Quarterly, 32(3), 253-260 (2015)

Marín, F.V., D.J. Inciarte, H.G. Hernández y R.C. Pitre, Estrategias de las Instituciones de Educación Superior para la Integración de las Tecnología de la Información y la Comunicación y de la Innovación en los Procesos de Enseñanza, Un Estudio en el Distrito de Barranquilla, Colombia, Formación Universitaria, 10(6), 29-38 (2017)

Martelo, R.J., A.L. Ponce y F. Acuña, Guía Metodológica para el Diseño de un Plan Estratégico Informático en Instituciones de Educación Superior, Formación Universitaria, 9(1), 91-98 (2016) 
MINTIC, Plan Vive Digital Colombia 2017 (2017)

Morales, M., J.M. Trujillo y F. Sánchez, Percepciones Acerca de la Integración de las TIC en el Proceso de Enseñanzaaprendizaje de la Universidad, Pixel-Bit, Revista de Medios y Educación, (46) (2015)

Niebles-Núñez, W.A., H.G. Hernández-Palma y D. Cardona-Arbeláez, Gestión Tecnológica del Conocimiento: Herramienta Moderna para la Gerencia de Instituciones Educativas, Revista de Investigación, Desarrollo e Innovación, 7(1), 25-36 (2016)

Owen, G.T., Qualitative Methods in Higher Education Policy Analysis: Using Interviews and Document Analysis, The Qualitative Report, 19(26), 1-19 (2014)

Prendes, M.P., Competencias TIC para la Docencia en la Universidad Pública Española: Indicadores y Propuestas para la Definición de Buenas Prácticas: Programa de Estudio y análisis, Informe del Proyecto EA2009-0133 de la Secretaría del Estado de Universidades e Investigación (2010)

Rajabi, A., M.J. Ghazali, J. Syarif y A.R. Daud, Development and Application of Tool Wear: A Review of the Characterization of TiC-based Cermets with Different Binders, Chemical Engineering Journal, 255, 445-452 (2014)

Ritchie, J., J. Lewis, C. Nicholls y R. Ormston, Qualitative Research Practice: A Guide for Social Science Students and Researchers, Sage (2013)

Rodríguez, J.L., Las TIC en Educación: Caminando Hacia las TAC, 3C TIC, 5(4), 55-62 (2016)

Said-Hung, E., F.I. Díaz-Granados y otros cinco autores, Fortalecimiento Pedagógico en las Universidades en Colombia a través de las TIC. Caso Región Caribe, Educación XX1, 18(2), 277-304 (2015)

Sikoyo, L., Contextual Challenges of Implementing Learner-centred Pedagogy: The Case of the Problem-solving Approach in Uganda, Cambridge Journal of Education, 40(3), 247-263 (2010)

Steffens, E., D. Ojeda y otros cuatro autores, Niveles de Pensamiento Crítico en Estudiantes de Universidades en Barranquilla (Colombia), Revista Espacios, 38 (30), 5-17 (2017)

Torres, J.M.P. y S.R Velandia, Influencia de las Estrategias Pedagógicas en los Procesos de Aprendizaje de los Estudiantes de una Institución de Básica Primaria de la Ciudad de Bucaramanga, Puente, 7(2), 117-130 (2017)

Valencia, R.E., Las TIC como Instrumento Pedagógico en la Educación Superior, RIDE Revista lberoamericana para la Investigación y el Desarrollo Educativo, 5(9), 64-79 (2015)

Vargas, A.D., Espacio de Investigación para la Educación de la Paz y la Convivencia Apoyado por las TIC, Opción, 32(8) (2016)

Vasović, N., y D. Milašinović, Modern Information and Communication Technologies in Higher Education, In $7^{\text {th }}$ International conference Higher Education in Function of Sustainable Development, Užice (2014)

Vila, R.R., S. Mengual-Andrés y C. L. A. Robles, ICT and New Educational Horizons in the Complex Society, In Education and Innovation in the University: Comparative Study between Italy and Spain, 239-251 (2016) 
